\title{
In vivo tumor targeting of gold nanoparticles: effect of particle type and dosing strategy
}

This article was published in the following Dove Press journal:

International Journal of Nanomedicine

5 March 2012

Number of times this article has been viewed

\author{
Priyaveena Puvanakrishnan' \\ Jaesook Park' \\ Deyali Chatterjee ${ }^{2}$ \\ Sunil Krishnan ${ }^{2}$ \\ James W Tunnell' \\ 'Department of Biomedical \\ Engineering, The University of Texas \\ at Austin, Austin, TX, USA; \\ ${ }^{2}$ The UT MD Anderson Cancer \\ Center, Houston, TX, USA
}

\begin{abstract}
Gold nanoparticles (GNPs) have gained significant interest as nanovectors for combined imaging and photothermal therapy of tumors. Delivered systemically, GNPs preferentially accumulate at the tumor site via the enhanced permeability and retention effect, and when irradiated with near infrared light, produce sufficient heat to treat tumor tissue. The efficacy of this process strongly depends on the targeting ability of the GNPs, which is a function of the particle's geometric properties (eg, size) and dosing strategy (eg, number and amount of injections). The purpose of this study was to investigate the effect of GNP type and dosing strategy on in vivo tumor targeting. Specifically, we investigated the in vivo tumortargeting efficiency of pegylated gold nanoshells (GNSs) and gold nanorods (GNRs) for single and multiple dosing. We used Swiss $n u / n u$ mice with a subcutaneous tumor xenograft model that received intravenous administration for a single and multiple doses of GNS and GNR. We performed neutron activation analysis to quantify the gold present in the tumor and liver. We performed histology to determine if there was acute toxicity as a result of multiple dosing. Neutron activation analysis results showed that the smaller GNRs accumulated in higher concentrations in the tumor compared to the larger GNSs. We observed a significant increase in GNS and GNR accumulation in the liver for higher doses. However, multiple doses increased targeting efficiency with minimal effect beyond three doses of GNPs. These results suggest a significant effect of particle type and multiple doses on increasing particle accumulation and on tumor targeting ability.
\end{abstract}

Keywords: gold nanorods, gold nanoshells, tumor targeting, multiple dosing

\section{Introduction}

Nanovectors are increasingly being used as tumor-targeting agents for improved disease diagnosis and treatment strategies. Nanoparticles' design, physical, and chemical properties are central to achieving effective tumor targeting and consequently improving cancer diagnosis and treatment. In vivo tumor targeting using nanoparticles has primarily been achieved using a passive targeting mechanism known as enhanced permeability and retention (EPR). ${ }^{1}$ The tumors' inherent leaky vasculature and ineffective lymph systems, enable systemically circulating nanoparticles to extravasate and accumulate in tumors due to the EPR effect. ${ }^{2}$ Recent efforts have focused on active targeting, which exploits the over-expression of surface receptors on cancer cells by providing targeting ligands that can engage these receptors. ${ }^{3-5}$ Choi et al demonstrated that targeted nanoparticles could provide greater intracellular delivery of therapeutic agents to the cancer cells within solid tumors than their nontargeted analogs. ${ }^{6}$ Although, there is continuous effort to identify suitable targeting moieties and ideal design 
parameters for active and passive targeting, respectively, little is known about the effect of nanoparticle dosing strategies on tumor targeting and accumulation capacity.

Multiple dosing is most commonly used in chemotherapy and radiation therapy for slowing and/or inhibiting tumor growth. ${ }^{7-9}$ Recently, Schluep et al demonstrated that a multiple dose of IT-101, a conjugate of camptothecin and a cyclodextrin-based polymer, showed increased antitumor activity in seven different mouse tumor xenografts. ${ }^{10}$ In addition, Herbst et al demonstrated high antitumor activity in patients with head and neck cancer using multiple doses of ZD1839 (gefinitib, an epidermal growth factor receptor tyrosine kinase inhibitor). ${ }^{11}$ In the present study, we investigated the effect of a multiple dosing strategy using pegylated gold nanoparticles to increase the in vivo tumor targeting competence.

Pegylated gold nanoparticles (GNPs) are ideal for in vivo use as they are stable, non-toxic, and possess unique optical and thermal properties that make them effective contrast and photo-thermal agents. ${ }^{12,13}$ The polyethylene glycol (PEG) coating on the gold nanoparticles' surface enables them to circulate longer and reduces nonspecific uptake by the reticuloendothelial system (RES). ${ }^{3}$ The RES, one of the body's filtration and defense mechanisms, functions to remove foreign particles and irregular red and white blood cells as well as opsonized particles. ${ }^{14}$ The liver and spleen that belong to the RES system are mainly responsible for the removal of different sized particles and hence these organs absorb a large percentage of systemically administered nanoparticles. ${ }^{15}$ Most of the biodistribution studies have shown that approximately $50 \%-60 \%$ of the injected dose of nanoparticles is taken up by the liver and spleen, respectively. ${ }^{16}$

Particles ranging from $10 \mathrm{~nm}$ to $15 \mu \mathrm{m}$ in size have different biodistribution and pharmacokinetic parameters. ${ }^{17}$ Experiments from animal models suggest that sub- $150 \mathrm{~nm}$ particles with neutral or slightly negatively charged entities can move through tumor tissue. ${ }^{18}$ Recent data show that systemically delivered nanoparticles in the $50-100 \mathrm{~nm}$ size range that carry a very slight positive charge can penetrate throughout large tumors. ${ }^{19}$ Therefore, nanoparticles in the $10-150 \mathrm{~nm}$ size range and with a neutral charge should have access to tumors when dosed into the circulatory system. ${ }^{20}$ Perrault et al have examined the particle size-dependent permeation of the tumor mass for the $20-100 \mathrm{~nm}$ size range and revealed a difference in behavior for different sizes, demonstrating that particle design has tremendous consequences on tumor targeting behavior. ${ }^{21}$
In this study, we investigated the effect of multiple dosing of GNPs and nanoparticle type to improve tumor-targeting efficiency. We utilized large pegylated gold nanoshells (GNSs) and small pegylated gold nanorods (GNRs) and compared the effect of the size of these particles on tumor targeting efficiency. Specifically, we compared the effect of single and multiple doses of GNRs and GNSs on in vivo tumor targeting. The choice of particles was driven by the ability of these particles to be photothermally activated. We used neutron activation analysis (NAA) to determine the amount of GNPs accumulated for the different doses and the percent accumulation of GNRs and GNSs in a squamous cell carcinoma tumor model. Our results indicated the efficacy of multiple dosing with increased accumulation of GNPs in tumors. Further, histopathological analysis of the tissues confirmed no acute toxicity due to multiple dosing. In addition, we studied the effect of particle type on tumor targeting and confirmed that smaller particles have enhanced accumulation in tumors compared to larger nanoparticles.

\section{Materials and methods Synthesis of pegylated gold nanoshells and gold nanorods GNS synthesis}

Nanoshells and nanorods used in this study were obtained from Nanospectra Biosciences Inc, (Houston, TX). The GNS fabrication is based on the method of Oldenburg. ${ }^{22}$ Briefly, gold colloids, $1-3 \mathrm{~nm}$ in diameter, were grown over an aminated $120 \pm 12 \mathrm{~nm}$ core of colloidal silica (Precision Colloids, LLC, Cartersville, GA). Gold colloid and the particles were then further reacted with $\mathrm{HAuCl}_{4}$ in the presence of formaldehyde causing the gold surface to grow and coalesce, ultimately forming a complete shell. The gold surface was then pegylated using thiolated polyethylene glycol (SH-PEG) (Laysan Bio, Huntsville, AL) to improve stability and blood circulation. GNS formation and dispersion in solution were assessed using a UV-Vis spectrophotometer. A concentration of $2.66 \times 10^{9}(100 \times$ dilution factor $)$ particles $/ \mathrm{mL}$ produced a peak OD of $\sim 1$. The particles were designed to have a core size of $120 \mathrm{~nm}$ and a shell thickness of $15 \mathrm{~nm}$ resulting in a peak Plasmon resonance at $810 \mathrm{~nm}$. For passive targeting, SH-PEG was added to the shell surface by combining $5 \mu \mathrm{M}$ SH-PEG and GNS in deionized water for 12 hours, followed by diafiltration to remove the excess SH-PEG. The GNS were in $1 \mathrm{~L}$ volume, at a concentration of approximately $4 \times 10^{9}$ particles $/ \mathrm{mL}$. This corresponds to a particle concentration of $\sim 7 \mathrm{pM}$ when $5 \mu \mathrm{M}$ PEG-SH is added. 


\section{GNR synthesis}

Nanorods were synthesized using the method developed by Jana et al..$^{23}$ Briefly, gold seed particles were prepared by adding $250 \mu \mathrm{L}$ of $10 \mathrm{mM} \mathrm{HAuCl}{ }_{4} 3 \mathrm{H}_{2} \mathrm{O}$ to $7.5 \mathrm{~mL}$ of $100 \mathrm{mM}$ cetyltrimethylammonium bromide with brief, gentle mixing. Freshly prepared $600 \mu \mathrm{L}$ of ice-cold $10 \mathrm{mM} \mathrm{NaBH}_{4}$ solution was added and the solution was mixed for 2 minutes. The nanorod growth solution was prepared by adding $40 \mathrm{~mL}$ of $100 \mathrm{mM}$ cetyltrimethylammonium bromide, $1.7 \mathrm{~mL}$ of $10 \mathrm{mM} \mathrm{HAuCl} \cdot 3 \mathrm{H}_{2} \mathrm{O}$, and $250 \mu \mathrm{L}$ of $10 \mathrm{mM} \mathrm{AgNO}$ followed by $270 \mu \mathrm{L}$ of $100 \mathrm{mM}$ ascorbic acid. To initiate nanorod growth, $840 \mu \mathrm{L}$ of the seed solution was added to the growth solution, mixed gently, and left still for 40 minutes. Excess reactants were removed by centrifugation and resuspension in deionized (DI) water. For in vivo applications, the GNRs were pegylated by the addition of $1 \mathrm{mM}$ thiolterminated methoxypolyethylene glycol (mPEG-SH) (Laysan Bio, Arab, AL) and the solution was stirred overnight. The final pegylated rod solution was cleaned by diafiltration of the solution into DI water. The synthesized GNRs had an aspect ratio of approximately 3.42 (GNR size: $24 \times 7 \mathrm{~nm}$ ). Both GNSs and GNRs were suspended in $10 \%$ trehalose because the particles exhibit greater long-term stability in trehalose compared to phosphate-buffered saline (PBS). In addition, $10 \%$ trehalose is iso-osmotic with blood. Figure 1 illustrates the extinction spectrum of GNSs and GNRs used in this study.

\section{Cell culture and tumor inoculation in mice}

A431 cells (CRL-1555, human epithelial carcinoma cell line, American Type Culture Collection [ATCC],

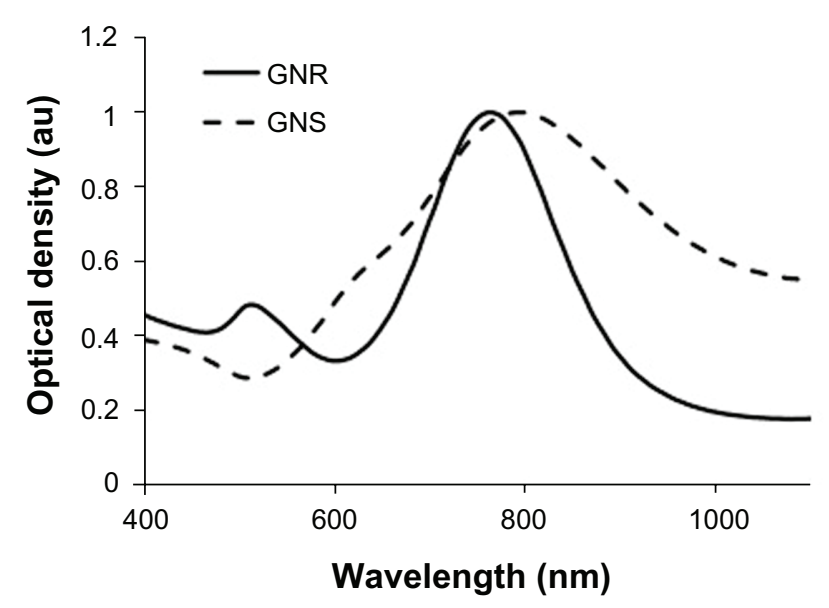

Figure I Extinction spectra of GNSs and GNRs. Abbreviations: GNS, gold nanoshells; GNR, gold nanorods.
Manassas, VA) were grown in Dulbecco's Modified Eagle's medium (30-2002, ATCC) with $10 \%$ fetal bovine serum $(30-2020, \mathrm{ATCC})$ at $37^{\circ} \mathrm{C}$ under $5 \% \mathrm{CO}_{2}$. When culture reached $90 \%$ confluency, the cells were detached from the flask by $0.25 \%$ trypsin-EDTA (30-2101, ATCC), centrifuged, and resuspended in sterile PBS. Four- to sixweek old nude mice (Swiss $n u / n u$ ) were inoculated with the A431 cells. Approximately $4 \times 10^{6}$ cells $/ 50 \mu \mathrm{L}$ were subcutaneously injected in the right thigh of 4- to 6-weekold nude mice (Swiss $n u / n u$ ). When tumors attained a size of $\sim 8-10 \mathrm{~mm}$ in diameter, we performed intravenous delivery of nanoparticles.

\section{Gold nanoparticle dosing: single and multiple dosing}

GNS and GNR solutions were intravenously injected via the mouse tail vein for single and multiple doses at 24-hour intervals. We systemically injected nanoparticles at 24 hour intervals since this time point has shown bulk nanoparticle clearance from blood allowing maximum particle accumulation in tumors. ${ }^{2,24}$ We distributed 38 mice into six different groups. Table 1 shows the animal use for a range of doses and both particle types. Group 1 and 2 animals received $7 \mu \mathrm{L} / \mathrm{g}$ of a single GNP dose (GNS: $2.74 \times 10^{8}$ particles $/ \mu \mathrm{L}$ and GNR: $2.00 \times 10^{11}$ particles $/ \mu \mathrm{L}$, optical density $=100$ ) and were sacrificed after 24 hours followed by tumor and organ extraction for analysis. Groups 3 and 4 and Groups 5 and 6 received multiple GNP doses once per day for 3 days and 5 days consecutively at 24-hour intervals, respectively. Following the three and five GNP doses, animals were sacrificed and the organs were extracted for analysis on days 4 and 6, respectively. Our control group consisted of three mice that received intravenous administration of trehalose.

\section{Neutron activation analysis (NAA)}

We performed neutron activation analysis (NAA) to quantify the amount of gold present in the extracted tumors and livers from the six mice groups. NAA is the gold standard method for trace gold quantification in biological samples, with sensitivities down to $70 \mathrm{pg} .{ }^{24} \mathrm{NAA}$ was performed in

Table I Number of animals utilized in each dosing group for GNS and GNR injections

\begin{tabular}{llll}
\hline Particle & \multicolumn{2}{l}{ Doses administered } \\
\cline { 2 - 4 } type & $\mathbf{I}$ & $\mathbf{3}$ & $\mathbf{5}$ \\
\hline GNS & Group I $(\mathrm{n}=7)$ & Group 3 $(\mathrm{n}=6)$ & Group 5 $(\mathrm{n}=6)$ \\
GNR & Group 2 $(\mathrm{n}=7)$ & Group 4 $(\mathrm{n}=6)$ & Group 6 $(\mathrm{n}=6)$ \\
\hline
\end{tabular}

Abbreviations: GNS, gold nanoshells; GNR, gold nanorods. 
the Nuclear Engineering Teaching Laboratory (NETL) at The University of Texas at Austin. Extracted tissues were weighed and placed inside the NAA vials. Following mass measurements, the vials containing tissue samples were placed in a desiccator and dehydrated for a week. Once desiccated, the vial lids were closed, and samples were sent to NETL for irradiation. From the amount of gold (mass of gold present in the samples) reported through NAA, and theoretical values for the mass of a single GNP, we calculated the number densities of GNSs and GNRs in the tumors and livers for all animal groups. The theoretically estimated mass of a single GNS (120 nm diameter core with $15 \mathrm{~nm}$ shell $)$ and GNR $(24 \times 7 \mathrm{~nm})$ were $1.33 \times 10^{-8} \mu \mathrm{g}$ and $1.61 \times 10^{-11} \mu \mathrm{g}\left(\rho_{\text {gold }}=19.32 \mathrm{~g} / \mathrm{cm}^{3}\right)$. Additionally, to compare the percent-injected dose for GNS and GNR, the gold mass present in the injected samples (standards) were determined using NAA. Standards of GNSs and GNRs were prepared in NAA vials similar to the tissue preparation and were sent to NETL for irradiation. Based on the number of particles injected (reported from NAA of standards) and the number of particles accumulated in the tumors and livers (reported from NAA of tissue samples) for a single dose, we calculated the percent-injected dose per gram of tissue for GNSs and GNRs.

\section{Histopathology}

We performed histopathological analysis to determine if there was acute toxicity in tumors and in the RES organs (liver and spleen) for the mice groups that received the highest GNP dose (five doses). Liver, spleen and tumor from groups 5 and 6 were examined by a pathologist at MD Anderson Cancer Center. The tissues from groups 1 and 2 (single dose) and the control group (trehalose injected) were also examined for comparison. In addition, we obtained light microscopy images of all the groups.

\section{Results}

\section{Accumulation of GNSs and GNRs in tumor for single and multiple dosing}

Figure 2 shows the accumulation of GNSs and GNRs in the tumor and liver for single, three and five doses based on NAA. These results show that three successive doses of GNPs lead to increased particle accumulation in the tumors. Specifically, three consecutive doses resulted in approximately 2 and
A

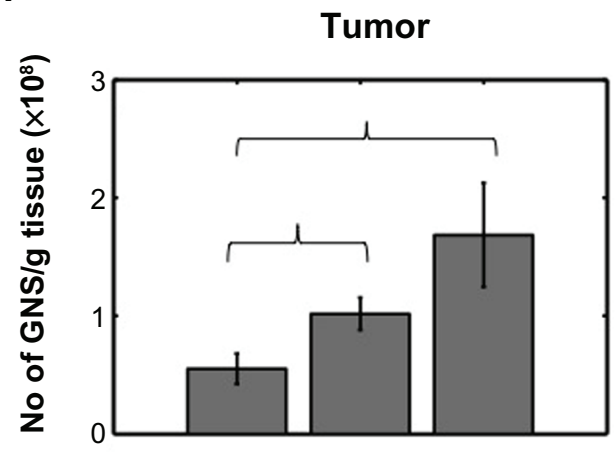

C

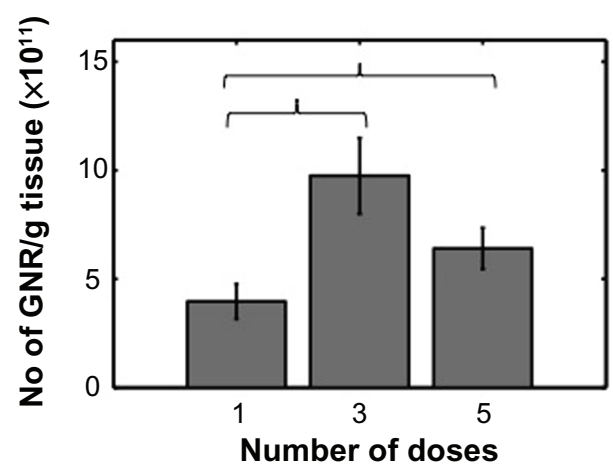

B

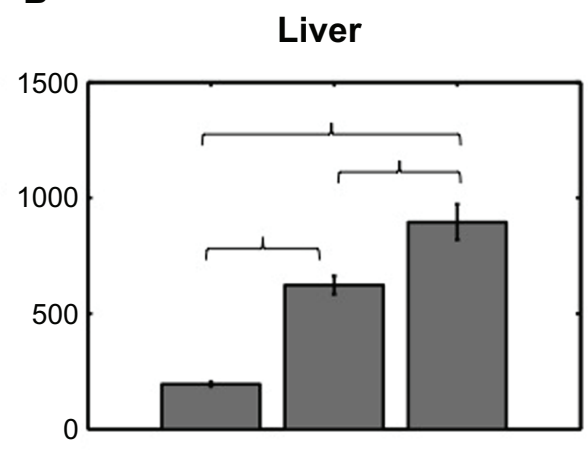

D

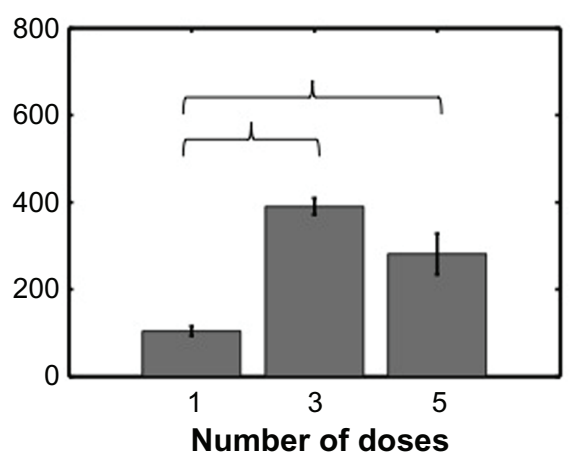

Figure 2 (A and B) Number density of GNSs/g of tissue for different numbers of doses (I,3, and 5) in tumor and liver, respectively. (C and D) Number density of GNRs/g of tissue for different doses in tumor and liver, respectively.

Notes: Error bars represent standard error. Brackets indicate statistical significance $P<0.05$.

Abbreviations: GNS, gold nanoshells; GNR, gold nanorods. 
2.45 times increase in particle accumulation with GNSs and GNRs, respectively (Figure 2A and C). However, particle accumulation in tumors receiving five consecutive doses of GNPs via the tail vein was less than expected. We observed only a 3-fold increase with GNSs and 1.6-fold increase with GNRs in tumors for five doses. Note that the increase we refer to here is relative to a single dose. In summary, we observed an increase in the number densities of GNSs and GNRs in the tumor for higher doses (three and five doses) compared to a single dose (Figure 2A and C). Specifically, one versus three doses and one versus five doses were statistically significant for both GNSs and GNRs. In the liver, which serves as a control, we observed a concomitant increase in number densities for GNSs with increase in doses: 3- and 4.6-fold increases for three and five doses, respectively (Figure 2B). However, for livers accumulated with GNRs, we observed an increase in number densities from single to three doses (3-fold increase) and a decrease from third to the fifth dose. However, this decrease/change was statistically insignificant. We also observed a similar, statistically insignificant decrease in the GNR tumor group.

\section{Effect of particle type on tumor uptake}

We observed a significant difference in tumor uptake of GNSs and GNRs for a single nanoparticle dose. Figure 3 illustrates the fraction of GNSs and GNRs accumulated in tumor and liver for a known injected particle dose. In the tumor, the smaller pegylated GNRs showed approximately twelve times higher accumulation $(1.35 \% \pm 0.29 \%$ injected dose per gram of tissue [ID/g tissue]) compared to the larger pegylated GNSs $(0.118 \% \pm 0.027 \% \mathrm{ID} / \mathrm{g}$ tissue $)$ after 24 hours. Similarly, we observed 15 and 6 times increase in percent accumulation for GNRs compared to GNSs at 72- and

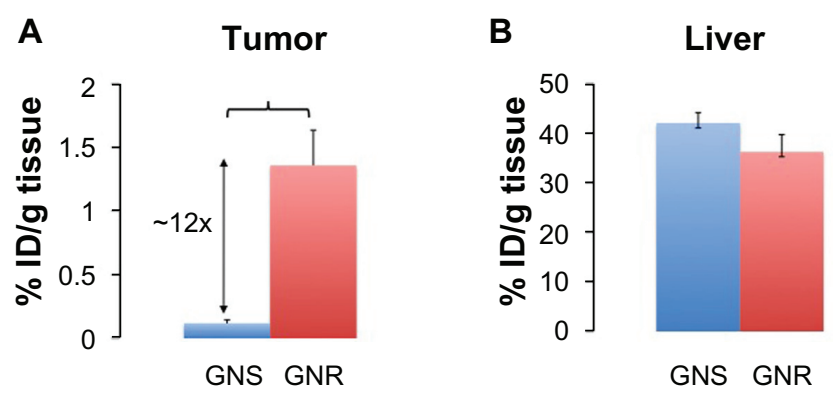

Figure 3 Percent injected dose per gram of tissue (\% ID/g of tissue) for GNRs and GNSs in (A) tumor and (B) liver 24 hours after intravenous injection. GNRs accumulated approximately twelve times higher than GNSs in the tumor.

Notes: Error bars represent standard error. Brackets indicate statistical significance $P<0.05$.

Abbreviations: GNS, gold nanoshells; GNR, gold nanorods; \% ID/g, percentage injected dose per gram. 120-hour time points, respectively (data not shown for 72 and 120 hours). However, in the liver, there was no statistical difference in the percent accumulation of GNSs and GNRs (GNSs: $42 \% \pm 2.11 \% \mathrm{ID} / \mathrm{g}$ tissue, GNRs: $37 \% \pm 3.4 \% \mathrm{ID} / \mathrm{g}$ tissue) at 24 hours.

\section{Histopathology of liver, spleen and tumor for multiple doses}

We observed no signs of acute toxicity such as sinusoidal dilation, inflammation or necrosis in the tumor, liver and spleen for multiple doses. Figure 4A-C show light microscopy images of tumor, liver and spleen stained with hematoxylin and eosin, respectively. The extent of typical inflammatory cell infiltration (lymphocytes and neutrophils) was similar in Group 1 tumors (single dose of GNSs and GNRs), Group 3 (five doses of GNSs and GNRs) and the control group (dose of trehalose). There were no significant differences in histopathological appearance observed in the livers (Figure 4B) and spleens (Figure 4C) between the groups. Furthermore, in the tumors, livers, and spleens analyzed, there was no histopathologically appreciable necrosis as a result of any presumed increase in microvascular blockade by aggregated GNPs, even in the group that received multiple doses of GNPs.

\section{Discussion}

In this study, we have shown that multiple dosing of gold nanoparticles injected systemically leads to a cumulative increase in particle accumulation in tumors. Multiple dosing is a common approach used in photodynamic, chemo and radiation therapies to treat tumors. In addition, multiple dosing has been shown to protract tumor growth in mouse models. In a recent study, mice bearing subcutaneous Neuro2A tumors were treated by intravenous injection with siRNA-containing nanoparticles formed with cyclodextrin-containing polycations (CDP). Three consecutive daily doses of transferrin (Tf)-targeted nanoparticles carrying two different siRNA sequences targeting ribonucleotide reductase subunit M2 (RRM2) led to slow tumor growth. ${ }^{25}$ The nanoparticles used in our present study are known to be effective in vivo tumor targeting agents. ${ }^{2,26}$ In addition, their strong NIR plasmon peak (Figure 1) enables them to scatter, absorb or luminesce when excited in the NIR. ${ }^{27,28}$ Recently, we demonstrated that systemically delivered GNSs passively accumulated in human colon tumors and served as excellent absorption-based contrast agents for wide-field tumor imaging. ${ }^{29}$

Smaller particles have high permeation/diffusion through tumor interstitium and correspondingly, higher clearance 


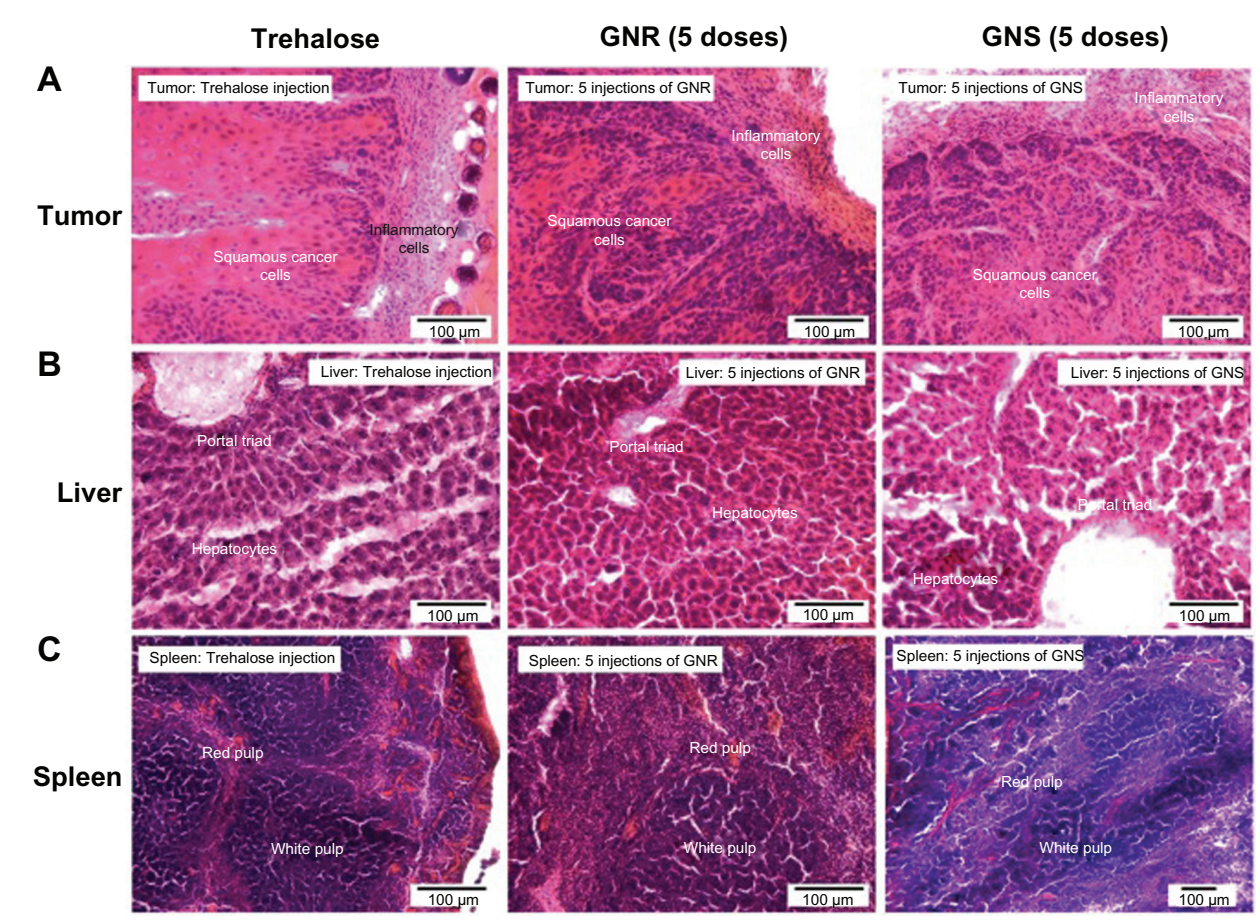

Figure 4 Light microscopy images of H\&E stained tumor, liver and spleen from mice that received five doses of GNSs and GNRs and a dose of trehalose (control). Abbreviations: GNS, gold nanoshells; GNR, gold nanorods; H\&E, hematoxylin and eosin.

to surrounding normal tissue (where they are likely to be cleared).$^{30}$ Jain et al and Boucher et al have shown that the interstitial fluid pressure decreases from the tumor core to the periphery and surrounding tissue, carrying nanoparticles with it by convection into the normal tissue as a function of particle size. ${ }^{31,32}$ This phenomenon could explain the decrease in smaller nanoparticle accumulation in tumors with days as noted in Figure 2C (decreasing trend in GNR number density observed in tumors from three to five doses). In contrast, larger particles have restricted and slow migration to the tumor environment along with slow clearance from the tumor owing to increased tumor accumulation with time. ${ }^{21,32}$ This explains the increase in GNS number density with increasing doses (more time to accumulate slowly) as seen in Figure 2A. However, a detailed study of the changes in nanoparticle accumulation in tumors for an extended time after single versus multiple dose delivery will help understand the mechanisms of uptake and clearance for various nanoparticle types.

We analyzed the livers of mice that received single and multiple dosing of both GNPs, since the liver has exhibited significantly higher nanoparticle accumulation compared to other RES organs. ${ }^{14}$ Although the liver and spleen are the main organs of the RES responsible for the removal of nanoparticles, the percentage accumulation in the liver is significantly higher compared to other organs such as the spleen, kidney, and brain. ${ }^{24}$ In addition, since the overall goal of the project did not encompass nanoparticle biodistribution throughout the body, we elected to process only the tumor and liver samples from all mice. The statistically significant increase in tumor accumulation of GNPs with increasing dose number in the liver (Figure 2B) validates the multiple dosing approach. However, we noticed an interesting trend in the GNR group. There was a drop-off in GNR accumulation from the three-dose group to the five-dose group (Figure 2D). Although uptake of particles from blood to liver may occur relatively quickly, excretion of these particles from the liver is relatively slow, often resulting in prolonged retention of GNPs. ${ }^{33}$ The decrease in GNR number density in the liver for the five-dose group, albeit statistically insignificant, is intriguing and warrants further investigation. A complete biodistribution study would definitely provide a better understanding of nanoparticle accumulation and clearance at higher doses.

Nanoparticles' size and half-life plays an important role in tumor accumulation of particles for diagnostic and therapeutic purposes. Perrault et al have shown that accumulation of smaller particles (less than $40 \mathrm{~nm}$ ) in tumors is a function of both size and half-life. For particles larger than $40 \mathrm{~nm}$, the accumulation is primarily a function of half-life. ${ }^{21}$ We used GNRs with a size of $24 \times 7 \mathrm{~nm}$ having an approximate halflife between 4-8 hours and GNSs of $135 \mathrm{~nm}$ diameter with 
an approximate half-life of 3 hours. ${ }^{2,24}$ The longer half-life and smaller size of GNRs explains their nearly 12-fold higher accumulation compared to the larger GNS.

With both particle types examined in this study, the highest tumor accumulation as a fraction of GNPs injected was only about $2(\% \mathrm{ID} / \mathrm{g})$. Hobbs et al have demonstrated that the vascular permeability and effective interstitial diffusion coefficient of a nanoparticle depends on the tumor type as well. ${ }^{34}$ The relatively low accumulation fraction of both pegylated GNRs and GNSs could be attributed to the tumor type as well. Pore cutoff sizes have been measured for a limited number of tumor models with the smallest reported at $100-200 \mathrm{~nm}^{34}$ To our knowledge, the pore cutoff size for the squamous cell carcinoma tumors (A431 tumors) used in this study has not yet been reported. The size of the GNPs did not affect the accumulation in livers as observed in Figure 3B. This correlates well with prior research showing that nanoparticle accumulation in the liver was independent of particle size. ${ }^{14}$ However, as noted in an earlier paragraph, the GNRs appear to clear much faster from the liver after 3 days.

Multiple dosing strategies did not lead to acute toxicity in mice during the course of the study. After administration of GNPs, mice were monitored daily for survival and behavior changes. There was no effect of multiple dose administrations of GNPs on mortality and behavioral impairment. There were no appreciable histopathological signs of acute toxicity such as inflammation, edema, sinusoidal dilation and necrosis in all mice groups. Further studies are required to ascertain the effect of multiple dosing on long-term clearance, safety and biodistribution. Although we have shown the effect of multiple dosing using passive targeting of pegylated GNPs, future work could include multiple dosing of actively targeted nanoparticles. Multiple dosing could potentially enhance imaging contrast and also improve thermal therapy efficacy.

\section{Conclusion}

We have demonstrated the safety and efficacy of a multiple dosing approach using GNPs and found it to increase particle accumulation in tumor. NAA data demonstrates that multiple dosing caused higher particle accumulation in tumors compared to a single dose. We did not notice any signs of acute toxicity in the tumor, liver or spleen with multiple doses of GNPs. We have shown that particle type affects tumor accumulation as well as clearance; smaller nanorods accumulated more within tumors than the larger nanoshells. These results suggest that multiple dosing might be an effective method to increase GNP accumulation in tumors.

\section{Acknowledgments}

The authors would like to thank Nanospectra Biosciences Inc, for providing the pegylated gold nanorods and nanoshells. The authors would like to thank Tracy Tipping in NETL for helping with irradiation of samples. The funding for this research was provided in part by the National Institutes of Health Grant No R01 CA132032 and R21 CA133691 and the American Society for Laser Medicine and Surgery.

\section{Disclosure}

The authors report no conflicts of interest in this work.

\section{References}

1. Maeda H, Fang J, Inutsuka T, Kitamoto Y. Vascular permeability enhancement in solid tumor: various factors, mechanisms involved and its implications. Int Immunopharmacol. 2003;3(3):319-328.

2. Goodrich G, Bao L, Gill-Sharp K, Sang K, Wang J, Payne JD. Photothermal therapy in a murine colon cancer model using nearinfrared absorbing gold nanorods. J Biomed Opt. 2010;15(1):018001.

3. Loo C, Lowery A, Halas N, West J, Crezek R. Immunotargeted nanoshells for integrated cancer imaging and therapy. Nano Lett. 2005;5(4): 709-711.

4. Sokolov K, Follen M, Aaron J, et al. Real-time vital optical imaging of precancer using anti-epidermal growth factor receptor antibodies conjugated to gold nanoparticles. Cancer Res. 2003;63(9): 1999-2004.

5. Loo C, Hirsch L, Lee MH, et al. Gold nanoshell bioconjugates for molecular imaging in living cells. Opt Lett. 2005;30(9):1012-1014.

6. Choi $\mathrm{CH}$, Alabi CA, Webster P, Davis ME. Mechanism of active targeting in solid tumors with transferrin-containing gold nanoparticles. Proc Natl Acad Sci U S A. 2010;107(3):1235-1240.

7. Williams KJ, Telfer BA, Stratford IJ, Wedge SR. ZD1839 ('Iressa'), a specific oral epidermal growth factor receptor-tyrosine kinase inhibitor, potentiates radiotherapy in a human colorectal cancer xenograft model. Br J Cancer. 2002;86(7):1157-1161.

8. Kato T, Sato K, Kakinuma H, Matsuda Y. Enhanced suppression of tumor growth by combination of angiogenesis inhibitor O-(chloroacetyl-carbamoyl)fumagillol (TNP-470) and cytotoxic agents in mice. Cancer Res. 1994;54(19):5143-5147.

9. Santini D, Vincenzi B, La Cesa A, et al. A new dose-intense epoetin alfa regimen effective in anemic cancer patients receiving chemotherapy: an open-label, non randomized, pilot study. Anticancer Res. 2005;25(1B):669-674.

10. Schluep T, Hwang J, Cheng J, et al. Preclinical efficacy of the camptothecin-polymer conjugate IT-101 in multiple cancer models. Clin Cancer Res. 2006;12(5):1606-1614.

11. Herbst RS, Maddox AM, Rothenburg ML, et al. Selective oral epidermal growth factor receptor tyrosine kinase inhibitor ZD1839 is generally well-tolerated and has activity in non-small-cell lung cancer and other solid tumors: results of a phase I trial. J Clin Oncol. 2002;20(18):3815-3825.

12. Hirsch LR, Stafford RJ, Bankson JA, et al. Nanoshell-mediated nearinfrared thermal therapy of tumors under magnetic resonance guidance. Proc Natl Acad Sci U SA. 2003;100(23):13549-13554.

13. Huang X, El-Sayed IH, Qian W, El-Sayed MA. Cancer cell imaging and photothermal therapy in the near-infrared region by using gold nanorods. J Am Chem Soc. 2006;128(6):2115-2120.

14. De Jong WH, Hagens WI, Krystek P, Burger MC, Sips AJ, Geertsma RE. Particle size-dependent organ distribution of gold nanoparticles after intravenous administration. Biomaterials. 2008;29(12):1912-1919. 
15. Terentyuk GS, Maslyakova GN, Suleymanove LV, et al. Circulation and distribution of gold nanoparticles and induced alterations of tissue morphology at intravenous particle delivery. J Biophotonics. 2009;2(5):292-302.

16. Melancon MP, Lu W, Yang Z, et al. In vitro and in vivo targeting of hollow gold nanoshells directed at epidermal growth factor receptor for photothermal ablation therapy. Mol Cancer Ther. 2008;7(6):1730-1739.

17. Wang J, Byrne JD, Napier ME, DeSimone JM. More effective nanomedicines through particle design. Small. 2011;7(14):1919-1931.

18. Nomura T, Koreeda N, Yamashita F, Takakura Y, Hashida M. Effect of particle size and charge on the disposition of lipid carriers after intratumoral injection into tissue-isolated tumors. Pharm Res. 1998;15(1):128-132.

19. Hu-Lieskovan S, Heidel JD, Bartlett DW, Davis ME, Triche TJ. Sequence-specific knockdown of EWS-FLI1 by targeted, nonviral delivery of small interfering RNA inhibits tumor growth in a murine model of metastatic Ewing's sarcoma. Cancer Res. 2005;65(19):8984-8992.

20. Davis ME, Chen ZG, Shin DM. Nanoparticle therapeutics: an emerging treatment modality for cancer. Nat Rev Drug Discov. 2008;7(9):771-782.

21. Perrault SD, Walkey C, Jennings T, Fischer HC, Chan WC. Mediating tumor targeting efficiency of nanoparticles through design. Nano Lett. 2009;9(5):1909-1915.

22. Oldenburg SJ, Averitt RD, Westcott SL, Halas NJ. Nanoengineering of optical resonances. Chem Phys Letters. 1998;288:243-247.

23. Jana NR, Geargart L, Murphy CJ. Wet chemical synthesis of high aspect ratio cylindrical gold nanorods. J Physl Chem B. 2001;105(19): 4065-4067.

24. James WD, Hirsch LR, West JL, O’Neal PD, Payne JD. Application of INAA to the build-up and clearance of gold nanoshells in clinical studies in mice. J Radio Nucl Chem. 2007;271:455-459.
25. Bartlett DW, Davis ME. Impact of tumor-specific targeting and dosing schedule on tumor growth inhibition after intravenous administration of siRNA-containing nanoparticles. Biotechnol Bioeng. 2008;99(4):975-985.

26. O’Neal DP, Hirsch LR, Halas NJ, Payne JD, West JL. Photo-thermal tumor ablation in mice using near infrared-absorbing nanoparticles. Cancer Lett. 2004;209(2):171-176.

27. Puvanakrishnan P, Diagaradjane P, Kazmi SM, Dunn AK, Krishnan S, Tunnell JW. Narrow band imaging of squamous cell carcinoma tumors using topically delivered anti-EGFR antibody conjugated gold nanorods. Lasers Surg Med. In press.

28. Park J, Estrada A, Schwartz JA, et al. Intra-organ biodistribution of gold nanoparticles using intrinsic two-photon induced photoluminescence. Lasers Surg Med. 2010;42(7):630-639.

29. Puvanakrishnan P, Park J, Diagaradjane P, et al. Near-infrared narrow band imaging of gold/silica nanoshells in tumors. J Biomed Opt. 2009;14(2):024044.

30. Jain RK. Transport of molecules, particles, and cells in solid tumors. Annu Rev Biomed Eng. 1999;1:241-263.

31. Jain Rk, Baxter LT. Mechanisms of heterogeneous distribution of monoclonal antibodies and other macromolecules in tumors: significance of elevated interstitial pressure. Cancer Res. 1988;48(24 Pt 1): 7022-7032.

32. Boucher Y, Baxter LT, Jain RK. Interstitial pressure gradients in tissueisolated and subcutaneous tumors: implications for therapy. Cancer Res. 1990;50(15):4478-4484.

33. Choi HS, Liu W, Misra P, et al. Renal clearance of quantum dots. Nat Biotechnol. 2007;25(10):1165-1170.

34. Hobbs SK, Monsky WL, Yuan R, et al. Regulation of transport pathways in tumor vessels: role of tumor type and microenvironment. Proc Natl Acad Sci U S A. 1998;95(8):4607-4612.
International Journal of Nanomedicine

\section{Publish your work in this journal}

The International Journal of Nanomedicine is an international, peerreviewed journal focusing on the application of nanotechnology in diagnostics, therapeutics, and drug delivery systems throughout the biomedical field. This journal is indexed on PubMed Central, MedLine, CAS, SciSearch $\AA$, Current Contents ${ }^{\circledR} /$ Clinical Medicine,

\section{Dovepress}

Journal Citation Reports/Science Edition, EMBase, Scopus and the Elsevier Bibliographic databases. The manuscript management system is completely online and includes a very quick and fair peer-review system, which is all easy to use. Visit http://www.dovepress.com/ testimonials.php to read real quotes from published authors. 